\title{
A pH-responsive smart surface for the continuous separation of oil/water/oil ternary mixtures
}

\author{
Guannan Ju, Mengjiao Cheng and Feng Shi
}

To handle the serious issue of increasing oil spill accidents, many strategies have been proposed to either clean spilt oil or separate water/oil mixture. Especially, superhydrophilic/underwater superoleophobic smart materials have recently shown advantages in overcoming problems of oil blocking and water barriers during conventional oil/water-separating process of oil-rich mixtures with superhydrophobic/superoleophilic materials. However, to the best of our knowledge, no prior reports have detailed smart materials with the wetting properties of superhydrophobic/superoleophilic that can be applied in continuous in situ separations of oil/water/oil ternary mixtures, which are common in practical oil spill cases. Herein, we describe the fabrication and efficacy of a pH-responsive smart device for continuous in situ separations of such oil/water/oil ternary mixtures without the need for ex situ treatments. In air, the superhydrophobic/superoleophilic surface of the device allowed dichloromethane to permeate through while preventing water from passing. The superhydrophilicity/underwater superoleophobicity of the device surface following alkaline treatments prevented the passage of hexane while allowing water to penetrate the device. NPG Asia Materials (2014) 6, e111; doi:10.1038/am.2014.44; published online 11 July 2014

\section{INTRODUCTION}

Oil pollution caused by either accidental oil spills or industrial disposal has become a severe environmental problem, particularly in marine ecosystems. Various oil/water-separating methods and materials have been developed to clean and recover spilt oil from marine environments, including physical diffusion, oil skimmers ${ }^{1}$ and mechanical recovery. ${ }^{2}$ Materials with extreme surface-wetting properties have drawn a great deal of attention due to their facile fabrication, high efficiency and flexible operation. Since the pioneering work of Jiang and co-workers ${ }^{3}$ various superhydrophobic/superoleophilic materials have been developed for oil/water separation of oil-rich mixtures, including porous polymer membranes, ${ }^{4}$ metal mesh, ${ }^{3,5-7}$ carbon nanotube network films ${ }^{8}$ and polymer-nanoparticle composites. ${ }^{9}$ Inspired by the oleophobicity of fish scales, several additional superhydrophilic/underwater superoleophobic materials have been proposed $^{10-16}$ in water-rich mixtures that allow water infiltration but prevent oil penetration when used underwater. These superhydrophilic/underwater superoleophobic materials ${ }^{17-19}$ may very well solve the problems of oil blocking that occurred in the system of water/oil with lower density than water during the separating process in conventional superhydrophobic/superoleophilic oil/water-separating materials. Although the above work describes materials suitable for passing either water or oil while excluding the other, the challenge of separating three-phase oil/water/oil mixtures in situ using the wetting properties of superhydrophobic/ superoleophilic materials still remains.
In addressing this issue, researchers have introduced smart surfaces that are capable of reversible transformations from superhydrophobicity to superhydrophilicity as oil/water-separating films in response to stimuli such as temperature, $\mathrm{pH}$ and light exposure. Jiang's group fabricated a temperature-responsive material that acts as a dual water/oil on-off switch, in which water permeates through a block copolymer-coated mesh. ${ }^{20}$ In contrast, oil cannot penetrate the material below a low critical solution temperature. These properties then reverse, that is, oil permeation is allowed while water permeation is not, above the low critical solution temperature. Wang and coworkers $^{21}$ developed a $\mathrm{pH}$-responsive smart surface that when underwater, allows oil infiltration while preventing water infiltration at $\mathrm{pH}$ 6.5. The same surface, however, blocks oil at $\mathrm{pH} 2.0{ }^{21}$ While promising, the above methods are not suitable for in situ oil/water separations because ex situ treatments are needed to induce changes in surface wettability. For this reason, Jiang and co-workers ${ }^{22}$ developed a photoinduced oil/water separation film composed of stainless-steel mesh coated with an aligned $\mathrm{ZnO}$ nanorod array. This mesh acted as a photoresponsive material for in situ oil/water separation. However, to the best of our knowledge, no prior reports have detailed smart materials that can be applied in continuous in situ separations of oil/water/oil ternary mixtures, which are common in practical oil spill cases. The current report describes the fabrication and efficacy of a $\mathrm{pH}$-responsive smart device for continuous in situ separations of such oil/water/oil ternary mixtures without the need for ex situ treatments. In the as-prepared device, the superhydrophobicity/superoleophilicity

State Key Laboratory of Chemical Resource Engineering \& Key Laboratory of Carbon Fiber and Functional Polymer, Ministry of Education, Beijing University of Chemical Technology, Beijing, China

Correspondence: Professor F Shi, State Key Laboratory of Chemical Resource Engineering \& Key Laboratory of Carbon Fiber and Functional Polymer, Ministry of Education, Beijing University of Chemical Technology, 15 Beisanhuan East Road, Chaoyang District, Beijing 100029, China.

E-mail: shi@mail.buct.edu.cn

Received 21 January 2014; revised 7 May 2014; accepted 8 May 2014 
of the active film allowed the bottom oil layer in a ternary mixture to infiltrate while the water layer was prevented from passing through the film. Permeation of the water layer through the device was activated by increasing the $\mathrm{pH}$ value of the water layer, resulting in an in situ transformation of surface wettability from superhydrophobic to superhydrophilic. Finally, the top oil layer was blocked by the superhydrophilic/underwater superoleophobic surface. In this way, the ternary oil/water/oil mixture could be sequentially separated through the device with no extra treatments. This method may provide a novel and facile strategy for separating complex oil/water/ oil mixtures in oil spill accidents.

\section{MATERIALS AND METHODS}

The following chemicals were used as supplied: 1-decanethiol $\left(\mathrm{SH}\left(\mathrm{CH}_{2}\right)_{9} \mathrm{CH}_{3}\right)$, 11-mercaptoundecanoic acid $\left(\mathrm{SH}\left(\mathrm{CH}_{2}\right)_{10} \mathrm{COOH}\right)$ from Sigma-Aldrich (St Louis, MO, USA), and $\mathrm{HF}(40 \%), \mathrm{AgNO}_{3}, \mathrm{HCl}$ and $\mathrm{NaOH}$ from Sinopharm Chemical Reagent Beijing Co., Ltd., Beijing, China. Copper foam was purchased from Anping Xinlong Wire Mesh Manufacture Co., Ltd., Anping, China. p-Doped silicon (111) wafers were obtained from GRINM Semiconductor Materials Co., Ltd., Beijing, China. Scanning electron micrographs were acquired on an EVO MA25 (Carl Zeiss, Wetzlar, Germany) at $20.0 \mathrm{kV}$. Photographic images were taken with a camera (D5000; Nikon, Tokyo, Japan). Contact angle measurements were performed on an OCA20 instrument (DataPhysics Instruments GmbH, Filderstadt, Germany).

Fabrication of the pH-responsive smart device. To make the smart device, a piece of porous copper foam was folded to a rectangular box without a top surface $(2.5 \mathrm{~cm} \times 2.5 \mathrm{~cm} \times 1.3 \mathrm{~cm})$. The device was cleaned in ethanol and deionized water, and silicon wafers were treated in a solution of $\mathrm{H}_{2} \mathrm{SO}_{4} / \mathrm{H}_{2} \mathrm{O}_{2}$ $(7: 3, \mathrm{v} / \mathrm{v})$. First, the rectangular box and a silicon wafer $(0.5 \mathrm{~cm} \times 0.5 \mathrm{~cm})$ were attached tightly with a binder clip and immersed in a mixture of aqueous $\mathrm{AgNO}_{3}(0.02 \mathrm{M})$ and $\mathrm{HF}(5 \mathrm{M})$ at $50{ }^{\circ} \mathrm{C}$ for $20 \mathrm{~min}$. Second, the as-prepared box was modified by immersion in a mixed ethanol solution of $\mathrm{SH}\left(\mathrm{CH}_{2}\right)_{9} \mathrm{CH}_{3}\left(0.6 \times 10^{-3} \mathrm{M}\right)$ and $\mathrm{SH}\left(\mathrm{CH}_{2}\right)_{10} \mathrm{COOH}\left(0.4 \times 10^{-3} \mathrm{M}\right)$ overnight. Finally, the device was washed with ethanol and deionized water, and dried in oven.

\section{RESULTS AND DISCUSSION}

As the copper foam is flexible, containers of arbitrary shapes and sizes can be easily folded or processed; to act as the smart device for oil/water separation, the copper foam was folded to a rectangular box without a top surface. The $\mathrm{pH}$-responsive surfaces of the device were obtained by combining electroless metal deposition with selfassembled monolayers. ${ }^{23-25}$ In the electroless deposition in aqueous solution of $\mathrm{AgNO}_{3}$ and $\mathrm{HF}$, the silicon wafer attached to the device is used to react with silver ions through galvanic cell reaction and thus promotes the reduction of silver ions to silver. ${ }^{26-28}$ Scanning electron microscopy images were acquired to evaluate the surface morphology of the smart device both before and after silver deposition. The untreated copper foam is porous with staggered holes of an average diameter of around $300 \mu \mathrm{m}$ (Figure 1a). The inset of Figure 1a shows the magnified copper mesh, which has a relatively low surface roughness. Figure $1 \mathrm{~b}$ indicates that after immersion in the silver solution, the porous foam was coated with a thick and compact film having few observable holes. The micrograph in the inset of Figure $1 \mathrm{~b}$ shows close-packed, microscale dendritic structures with three-dimensional nano-branches. Energy dispersive X-ray data (Supplementary Figure S1) and X-ray photoelectron spectroscopy (Supplementary Figure S2) showed that these structures were silver aggregates. The coverage of these silver aggregates on the porous foam depended largely on the deposition time. Long deposition times can result in a fragile device. In its current form, the hierarchical microand nanostructure of the millimeter-scale copper foam was sufficient to provide sufficient surface roughness for the fabrication of a
$\mathrm{pH}$-responsive smart surface presenting wettability transformation between superhydrophobicity/superhydrophilicity after further modification with mixed thiols of $\mathrm{SH}\left(\mathrm{CH}_{2}\right)_{9} \mathrm{CH}_{3}$ and $\mathrm{SH}\left(\mathrm{CH}_{2}\right)_{10} \mathrm{COOH}$ at a molar ratio of $4: 6 .{ }^{29-31}$ The surface modification of the mixed thiols was confirmed through X-ray photoelectron spectroscopyspectra (Supplementary Figure S2). At the ratio of 4:6, the modified rough surface will exhibit superhydrophobicity under acidic conditions because the carboxylic acid groups are mostly protonated, and thus the methyl groups play a dominant role in surface wettability; under alkaline condition, the majority of carboxylic acid groups is deprotonated and hydrated, contributing to a superhydrophilic surface property.

To verify the differential surface wettability in air and underwater at different $\mathrm{pH}$ values, aqueous or oil droplets were applied to the asprepared films both in air and underwater. Figure 1c shows photographs of the device surface operating in air/liquid/solid contact mode. The upper photographs display the initial states when acidic, neutral and alkaline droplets were placed on the surface, respectively. With time, the alkaline droplet spread completely over the surface, while the acidic and neutral droplets remained unchanged, as shown in the bottom images. This phenomenon can be interpreted as follows. Under acidic or neutral conditions of air/liquid/solid contact, the carboxylic acid groups of the mixed thiols are mostly protonated and the wettability of the surface is dominated by the methyl groups, resulting in a superhydrophobic surface. In contrast, with an alkaline droplet, the majority of carboxylic acid groups are deprotonated, resulting in an easily hydrated, superhydrophilic surface. To assess underwater wettability, the as-prepared film was pressed into an acidic solution with tweezers because the porosity and the superhydrophobicity of the copper foam makes it more likely to store air or capture bubble layers when immersed in pure water (Figure 1d), resulting in a lower density than that of water. Dyed dichloromethane, an oil simulant, was then injected underwater through a dropper onto the device surface. Upon contact, the oil droplet was quickly absorbed. Figure 1d shows that the superhydrophobicity/superoleophilicity of the device was well retained underwater under acidic conditions. To induce a switch in surface-wetting properties, the film was then immersed in an alkaline solution, which completely wet the film with no observable bubble layer (Figure 1e). Furthermore, an identical oil droplet held its round shape when injected onto the surface underwater, indicating underwater superoleophobicity. Thus, the as-prepared smart surface underwent a transformation from superhydrophobicity/superoleophilicity to superhydrophilicity/underwater superoleophobicity in response to the $\mathrm{pH}$ of the surrounding solution. The above results were further supported by contact angle measurements both in air and underwater (Supplementary Figures S3 and S4).

After fabricating the smart device and demonstrating a $\mathrm{pH}$ responsive switch in surface wettability, the device was evaluated with regard to oil/water separation. An oil/water mixture was constructed by adding $10 \mathrm{ml}$ of neutral water into $10 \mathrm{ml}$ of green-dyed dichloromethane, forming separate upper and bottom layers composed of water and oil phases, respectively. The as-prepared rectangular smart device was positioned over the top of a beaker, and the upper water layer of the mixture was poured into the interior of the device. No leakage of water was observed in the supportive beaker, which was due to the superhydrophobic surface of the smart device (Figure 2a). The green oil layer was then added to the device, which still contained the water layer. The superoleophilic properties of the device (Figure 2b) allowed the oil to pass quickly through the device and into the supporting beaker. In this way, oil can be quickly and 
a

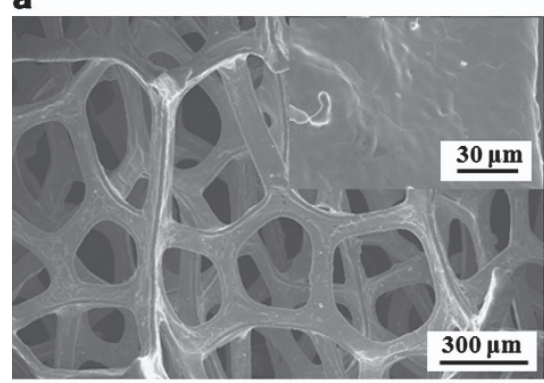

c

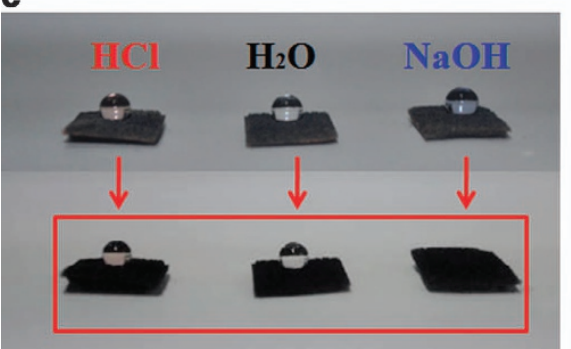

b

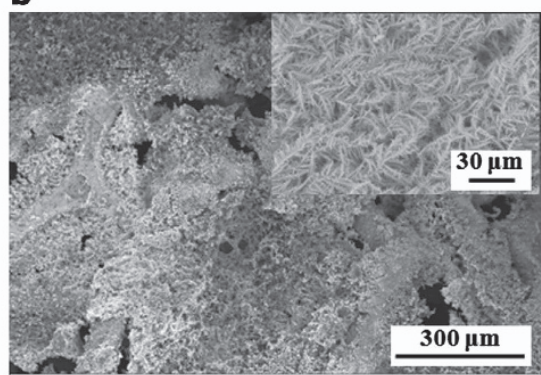

d

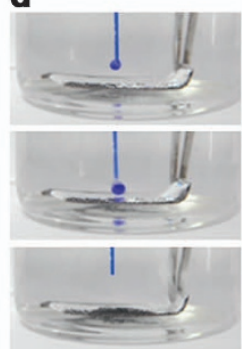

e

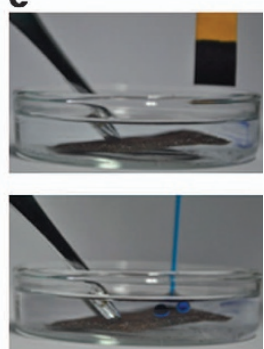

Figure 1 Scanning electron micrographs of copper foam (a) before and (b) after immersion in $\mathrm{HF} / \mathrm{AgNO}_{3}$ solution. The insets of a and $\mathbf{b}$ show local high magnification. (c) Photographs display the original states of droplets of $\mathrm{HCl}, \mathrm{H}_{2} \mathrm{O}$, and $\mathrm{NaOH}$ on the as-prepared smart surface (top images). After several

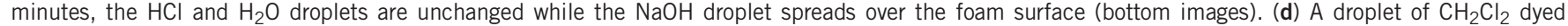
blue is injected underwater onto the as-prepared foam and absorbed immediately upon contact. (e) In alkaline solution, the foam is wetted and the oil droplet stands round on the surface.
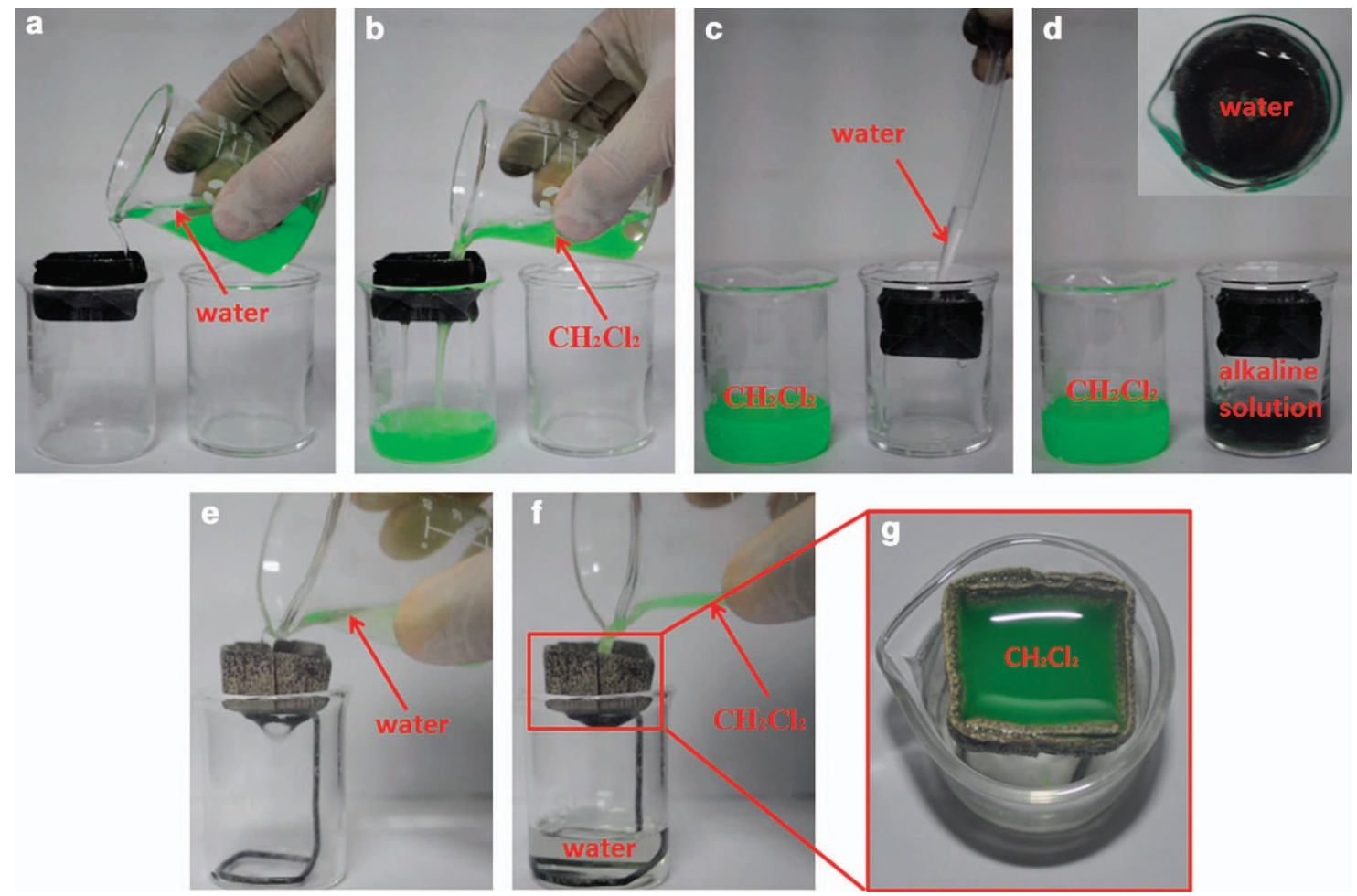

Figure 2 Photographs show the oil/water separation using the smart device. (a, b) A mixture of water and green-dyed oil is poured into the smart device; the oil passes through into the supporting beaker. (c) Water is prevented from infiltrating the superhydrophobic surface and remains in the device box. (d) An aqueous alkaline solution, however, permeates through the device. (e-g) Water passes through the alkaline-treated device while oil is blocked. 

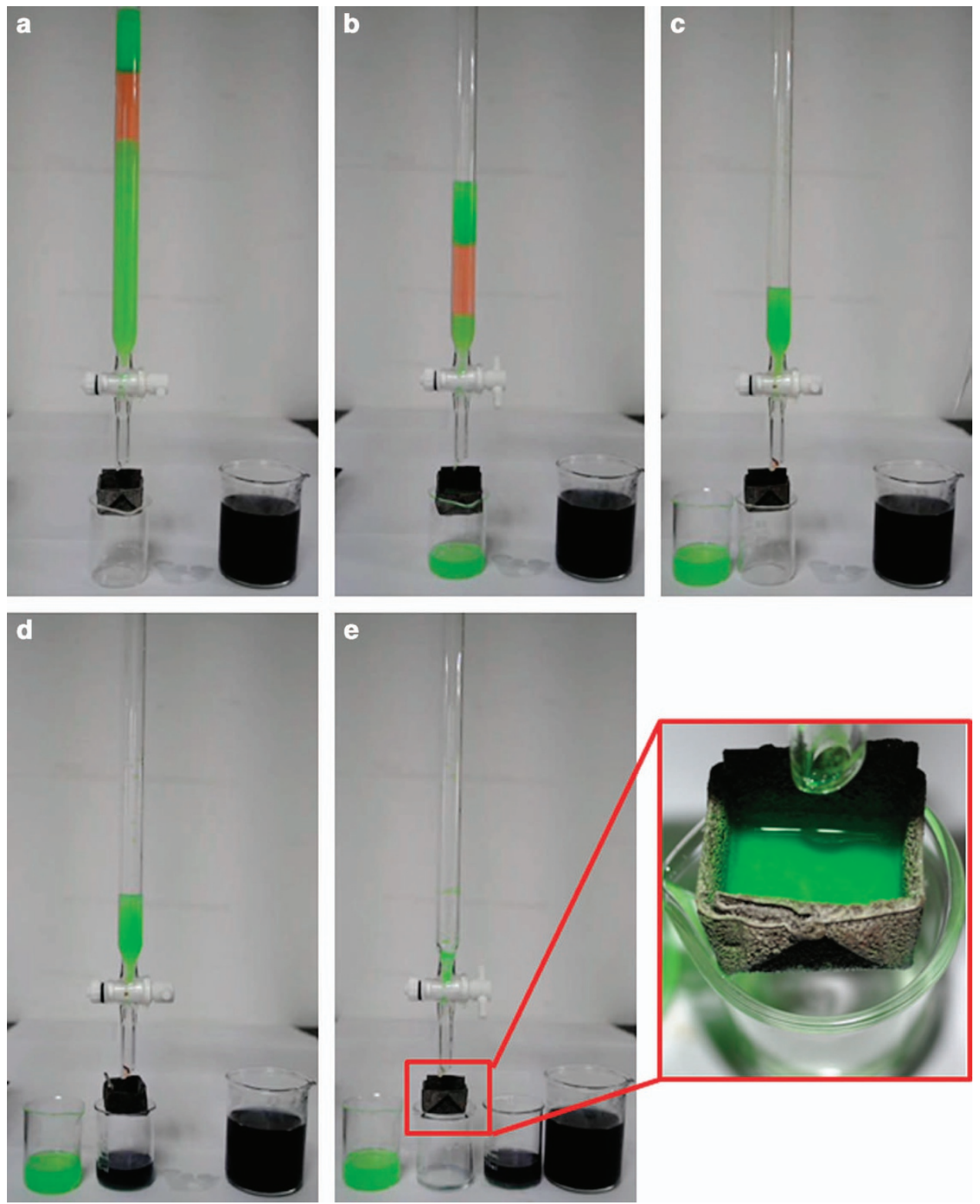

Figure 3 Photographs show oil/water/oil separation using the smart device. (a) A ternary mixture of hexane/acidic water/dichloromethane was added to a separatory funnel. The oil layer was dyed green, and the $\mathrm{pH}$ of the aqueous phase is indicated by litmus dye. (b) The bottom oil was released through the device. (c) The acidic aqueous solution was held within the device. (d) After adjusting the pH from acidic to alkaline, the water layer permeated through the device. (e) Due to underwater superoleophobicity, the upper oil layer was collected in the smart device and did not pass through.

efficiently separated from an oil/water mixture. Neutral water is effectively blocked by the film and remains in the device, as shown in Figure $2 \mathrm{c}$ and in the inset of Figure $2 \mathrm{~d}$. To release the water from the device, the $\mathrm{pH}$ of the water was adjusted to alkaline conditions, thereby inducing a switch in the surface properties of the device from superhydrophobic to superhydrophilic. Soon after the solution was changed to alkaline, as indicated by a litmus test, the water passed through the device into the supporting beaker. This transformation in wettability corresponds well with the results in Figure 1c. By taking advantage of the difference in wettability between oil and water and the $\mathrm{pH}$-responsive wettability of the modified copper foam, we succeeded in sequentially separating an oil/water mixture and collected the two phases in separate containers. The purity of the separated oil was measured with a water titrator to detect the water content within oil, which was tested to be w.t. $0.086 \%$.

The sequence of the separation can also be reversed. That is, water can be removed from the oil/water mixture first by initially increasing the alkalinity of the aqueous phase. Figure $2 \mathrm{e}$ shows the device supported by a scaffold of iron wire in a beaker and treated with an alkaline solution. As an identical oil/water mixture was poured into the device, colorless water permeated through the bottom surface and gradually gathered in the beaker. No dyed oil was observed in the beaker (Figure 2f). The oil phase of the mixture was prevented from passing through the smart surface and remained in the device, 
indicative of the superhydrophilic/underwater superoleophobic property of the device surface. This is in accordance with the results in Figure 1e. The top view of the separated oil and water phases in Figure $2 \mathrm{~g}$ shows that water was collected in the beaker while oil was held in the smart device, resulting in complete separation of the original oil/water mixture. These results demonstrate that the superhydrophilicity/underwater superoleophobicity can be used for effective oil/water separation.

The integration of the individual oil/water and water/oil separating procedures into a single process, which would be useful in practical separations of complex oil/water/oil ternary mixtures, was then evaluated. Two oils, hexane and dichloromethane, with densities below and above that of water, respectively, were selected. The oils were dyed green, and litmus dye was used to indicate the $\mathrm{pH}$ of the water layer. When the oils were mixed with acidic water in a separatory funnel, they formed oil/water/oil ternary layers as displayed in Figure 3a. The as-prepared smart device, supported by a beaker, was placed at the outlet of the funnel and a beaker of alkaline solution, dyed blue by litmus, was used for $\mathrm{pH}$ adjustment. Opening the valve on the separatory funnel released the bottom heavy oil, which quickly permeated through the smart device due to its superhydrophobicity/superoleophilicity (Figure 3b). The pink acidic solution was then released into the device. No aqueous solution was observed passing through the device and into the beaker (Figure 3c). The $\mathrm{pH}$ of the aqueous solution was then changed from acidic (pink) to alkaline (dark blue). As expected, this alkaline treatment transformed the device surface from superhydrophobic to superhydrophilic, resulting in rapid infiltration of the aqueous solution through the device and into the supporting beaker as shown in Figure 3d. Finally, the light oil layer was collected in the device (Figure $3 \mathrm{e}$ ). The above oil/water/oil separation was performed continuously and each component of the ternary mixture was collected individually. Note that no additional ex situ treatments were required throughout the entire separation. Thus, a pH-responsive transformation of the as-prepared smart device realized the sequential separation and collection of a complex ternary oil/water/oil mixture.

\section{CONCLUSION}

To summarize, a smart device was fabricated through electroless metal deposition followed by surface modification with a mixed thiol containing carboxylic groups and methyl groups. This modification allowed the surface wettability to be switched from superhydrophobic to superhydrophilic in response to the $\mathrm{pH}$ of an aqueous solution. The surface wettability of the smart device was investigated both in air and underwater under alkaline conditions, whereby superhydrophobicity/superoleophilicity and superhydrophilic/underwater superoleophobic properties were observed, respectively. By taking advantage of this $\mathrm{pH}$-dependant wettability, the smart device was applied to oil/water separations both in air and underwater. Efficient separation and collection of the individual components of a complex oil/water/ oil mixture was realized in a continuous process with no ex situ treatments. The continuous in situ separation of oil/water/oil ternary mixtures may provide a novel strategy for handling oil spills under various conditions and situations.

\section{ACKNOWLEDGEMENTS}

This work was supported by NSFC (21374006), the Program of the CoConstruction with Beijing Municipal Commission of Education of China, Program for New Century Excellent Talents in University (NCET-10-0211), the Fok Ying Tung Education Foundation (131013), Open Project of State Key
Laboratory of Supramolecular Structure and Materials (SKLSSM201401) and Beijing Young Talents Plan (YETP0488).

1 Fingas, M. Oil spills and their cleanup. Chem. Ind. 24, 1005-1008 (1995).

2 Konishi, M., Kishimoto, M., Tamesui, N., Omasa, T., Shioya, S. \& Ohtake, H. The separation of oil from an oil-water-bacteria mixture using a hydrophobic tubular membrane. Biochem. Eng. J. 24, 49-54 (2005).

3 Feng, L., Zhang, Z. Y., Mai, Z. H., Ma, Y. M., Liu, B. Q., Jiang, L. \& Zhu, D. B. A super-hydrophobic and super-oleophilic coating mesh film for the separation of oil and water. Angew. Chem. Int. Ed. 43, 2012-2014 (2004).

4 Zhang, J. L., Huang, W. H. \& Han, Y. C. A composite polymer film with both superhydrophobicity and superoleophilicity. Macromol. Rapid Commun. 27, 804-808 (2006).

5 Tian, D. L., Zhang, X. F., Wang, X., Zhai, J. \& Jiang, L. Micro/nanoscale hierarchical structured $\mathrm{ZnO}$ mesh film for separation of water and oil. Phys. Chem. Chem. Phys. 13, 14606-14610 (2011).

6 Wu, J., Chen, J., Qasim, K., Xia, J., Lei, W. \& Wang, B. P. A hierarchical mesh film with superhydrophobic and superoleophilic properties for oil and water separation. J. Chem. Technol. Biotechnol. 87, 427-430 (2012).

7 Gao, C. R., Sun, Z. X., Li, K., Chen, Y. N., Cao, Y. Z., Zhang, S. Y. \& Feng, L. Integrated oil separation and water purification by a double-layer $\mathrm{TiO}_{2}$-based mesh. Energy Environ. Sci. 6, 1147-1151 (2013).

8 Shi, Z., Zhang, W. B., Zhang, F., Liu, X., Wang, D., Jin, J. \& Jiang, L. Ultrafast separation of emulsified oil/water mixtures by ultrathin free-standing single-walled carbon nanotube network films. Adv. Mater. 25, 2422-2427 (2013).

9 Yang, J., Zhang, Z. Z., Xu, X. H., Zhu, X. T., Men, X. H. \& Zhou, X. Y. Superhydrophilicsuperoleophobic coatings. J. Mater. Chem. 22, 2834-2837 (2012).

10 Zhang, L. B., Zhong, Y. J., Cha, D. Y. \& Wang, P. A self-cleaning underwater superoleophobic mesh for oil-water separation. Sci. Rep. 3, 2326 (2013).

11 Xu, L. P., Zhao, J., Su, B., Liu, X. L., Peng, J. T., Liu, Y. B., Liu, H. L., Yang, G., Jiang, L., Wen, Y. Q., Zhang, X. J. \& Wang, S. T. An ion-induced low-oil-adhesion organic/inorganic hybrid film for stable superoleophobicity in seawater. Adv. Mater. 25, 606-611 (2013).

12 Liu, X. L., Zhou, J., Xue, Z. X., Gao, J., Meng, J. X., Wang, S. T. \& Jiang, L. Clam's shell inspired high-energy inorganic coatings with underwater low adhesive superoleophobicity. Adv. Mater. 24, 3401-3405 (2012).

13 Xue, Z. X., Wang, S. T., Lin, L., Chen, L., Liu, M. J., Feng, L. \& Jiang, L. A novel superhydrophilic and underwater superoleophobic hydrogel-coated mesh for oil/water separation. Adv. Mater. 23, 4270-4273 (2011).

14 Liu, M. J., Wang, S. T., Wei, Z. X., Song, Y. L. \& Jiang, L. Bioinspired design of a superoleophobic and low adhesive water/solid interface. Adv. Mater. 21, 665-669 (2009).

15 Cai, Y., Lin, L., Xue, Z. X., Liu, M. J., Wang, S. T. \& Jiang, L. Filefish-inspired surface design for anisotropic underwater oleophobicity. Adv. Funct. Mater. 24, 809-816 (2014).

16 Zhang, F., Zhang, W. B., Shi, Z., Wang, D., Jin, J. \& Jiang, L. Nanowire-haired inorganic membranes with superhydrophilicity and underwater ultralow adhesive superoleophobicity for high-efficiency oil/water separation. Adv. Mater. 25, 4192-4198 (2013).

17 Gao, X. F., Xu, L. P., Xue, Z. X., Feng, L., Peng, J. T., Wen, Y. Q., Wang, S. T. \& Zhang, X. J. Dual-scaled porous nitrocellulose membranes with underwater superoleophobicity for highly efficient oil/water separation. Adv. Mater. 26, 1771-1775 (2014).

18 Jin, M. H., Li, S. S., Wang, J., Xue, Z. X., Liao, M. Y. \& Wang, S. T. Underwater superoleophilicity to superoleophobicity: role of trapped air. Chem. Commun. 48, 11745-11747 (2012).

19 Xu, L. P., Peng, J. T., Liu, Y. B., Wen, Y. Q., Zhang, X. J., Jiang, L. \& Wang, S. T. Nacre-inspired design of mechanical stable coating with underwater superoleophobicity. ACS Nano 7, 5077-5083 (2013)

20 Xue, B. L., Gao, L. C., Hou, Y. P., Liu, Z. W. \& Jiang, L. Temperature controlled water/oil wettability of a surface fabricated by a block copolymer: application as a dual water/oil on-off switch. Adv. Mater. 25, 273-277 (2013).

21 Zhang, L. B., Zhang, Z. H. \& Wang, P. Smart surfaces with switchable superoleophilicity and superoleophobicity in aqueous media: toward controllable oil/water separation. NPG Asia Mater. 4, e8 (2012).

22 Tian, D. L., Zhang, X. F., Tian, Y., Wu, Y., Wang, X., Zhai, J. \& Jiang, L. Photo-induced water-oil separation based on switchable superhydrophobicity-superhydrophilicity and underwater superoleophobicity of the aligned $\mathrm{ZnO}$ nanorod array-coated mesh films. J. Mater. Chem. 22, 19652-19657 (2012).

23 Cheng, M. J., Gao, Y. F., Guo, X. P., Shi, Z. Y., Chen, J. F. \& Shi, F. A functionally integrated device for effective and facile oil spill cleanup. Langmuir 27, 7371-7375 (2011).

24 Cheng, M. J., Ju, G. N., Jiang, C., Zhang, Y. J. \& Shi, F. Magnetically directed clean-up of underwater oil spills through a functionally integrated device. J. Mater. Chem. A 1, 13411-13416 (2013).

25 Gao, Y. F., Cheng, M. J., Wang, B. L., Feng, Z. G. \& Shi, F. Diving-surfacing cycle within a stimulus-responsive smart device towards developing functionally cooperating systems. Adv. Mater. 22, 5125-5128 (2010).

26 Song, Y. Y., Gao, Z. D., Kelly, J. J. \& Xia, X. H. Galvanic deposition of nanostructured noble-metal films on silicon. Electrochem. Solid-State Lett. 8, C148-C150 (2005). 
27 Shi, F., Song, Y. Y., Niu, J., Xia, X. H., Wang, Z. Q. \& Zhang, X. Facile method to fabricate a large-scale superhydrophobic surface by galvanic cell reaction. Chem. Mater. 18, 1365-1368 (2006).

28 Peng, K. Q., Yan, Y. J., Gao, S. P. \& Zhu, J. Synthesis of large-area silicon nanowire arrays via self-assembling nanoelectrochemistry. Adv. Mater. 14, 1164-1167 (2002)

29 Ju, G. N., Cheng, M. J., Xiao, M., Xu, J. M., Pan, K., Wang, X., Zhang, Y. J. \& Shi, F. Smart transportation between three phases through a stimulus-responsive functionally cooperating device. Adv. Mater. 25, 2915-2919 (2013).

30 Cheng, M. J., Liu, Q., Ju, G. N., Zhang, Y. J., Jiang, L. \& Shi, F. Bell-shaped superhydrophilic-superhydrophobic-superhydrophilic double transformation on a pH-responsive smart surface. Adv. Mater. 26, 306-310 (2014).

31 Xiao, M., Cheng, M. J., Zhang, Y. J. \& Shi, F. Combining the marangoni effect and the $\mathrm{pH}$-responsive superhydrophobicity-superhydrophilicity transition to biomimic the locomotion process of the beetles of genus stenus. Small 9, 2509-2514 (2013).

(c) (1) (2) (2) This work is licensed under a Creative Commons Attribution-NonCommercial-ShareAlike 3.0 Unported License. The images or other third party material in this article are included in the article's Creative Commons license, unless indicated otherwise in the credit line; if the material is not included under the Creative Commons license, users will need to obtain permission from the license holder to reproduce the material. To view a copy of this license, visit http://creativecommons.org/licenses/by-nc-sa/3.0/

Supplementary Information accompanies the paper on the NPG Asia Materials website (http://www.nature.com/am) 